BMJ Paediatrics Open

\section{Weaning strategies for the withdrawal of non-invasive respiratory support applying continuous positive airway pressure in preterm infants: a systematic review and meta-analysis}

To cite: van Delft $B$, Van Ginderdeuren F, Lefevere J, et al. Weaning strategies for the withdrawal of non-invasive respiratory support applying continuous positive airway pressure in preterm infants: a systematic review and metaanalysis. BMJ Paediatrics Open 2020;4:e000858. doi:10.1136/ bmjpo-2020-000858

- Additional material is published online only. To view, please visit the journal online (http://dx.doi.org/10.1136/ bmjpo-2020-000858).

Received 31 August 2020 Revised 21 October 2020 Accepted 27 October 2020
Check for updates

(c) Author(s) (or their employer(s)) 2020. Re-use permitted under CC BY-NC. No commercial re-use. See rights and permissions. Published by BMJ.

${ }^{1}$ Neonatology, UZ Brussel, Brussels, Belgium

${ }^{2}$ Rehabilitation Research, Department of Physiotherapy, UZ Brussel, Brussels, Belgium ${ }^{3}$ Neonatology, UZ Brussel, Jette, Belgium

Correspondence to Dr Brenda van Delft; vandelftbrenda@gmail.com

\section{ABSTRACT}

Background The optimal method to wean preterm infants from non-invasive respiratory support (NIVRS) with nasal continuous positive airway pressure (CPAP) or highflow nasal cannula is still unclear, and methods used vary considerably between neonatal units.

Objective Perform a systematic review and metaanalysis to determine the most effective strategy for weaning preterm infants born before 37 weeks' gestation from NIVRS.

Method EMBASE, MEDLINE, CINAHL, Google and Cochrane Central Register of Controlled Trials were searched for randomised controlled trials comparing different weaning strategies of NIVRS in infants born before 37 weeks' gestation.

Results Fifteen trials (1.547 infants) were included. With gradual pressure wean, the relative risk of successful weaning at the first attempt was $1.30(95 \% \mathrm{Cl} 0.93$ to $1.83)$, as compared with sudden discontinuation. Infants were weaned at a later postmenstrual age (PMA) (median difference (MD) 0.93 weeks ( $95 \% \mathrm{Cl} 0.19$ to 1.67)). A stepdown strategy to nasal cannula resulted in an almost 3-week reduction in the PMA at successful weaning (MD $-2.70(95 \% \mathrm{Cl}-3.87$ to -1.52$))$ but was associated with a significantly longer duration of oxygen supplementation (MD 7.80 days (95\% $\mathrm{Cl} 5.31$ to 10.28)). A strategy using interval training had no clinical benefits. None of the strategies had any effect on the risk of chronic lung disease or the duration of hospital stay.

Conclusion A strategy of gradual weaning of airway pressure might increase the chances of successful weaning. Stepdown strategy from CPAP to nasal cannula is a useful alternative resulting in an earlier weaning, but the focus should remain on continued weaning in order to avoid prolonged oxygen supplementation. Interval training should probably not be used.

\section{INTRODUCTION}

Non-invasive respiratory support (NIVRS) is widely used for the management of respiratory disorders in preterm infants. Common indications are neonatal respiratory distress syndrome (RDS), apnoea of prematurity,

\section{What is known about the subject?}

Non-invasive respiratory support (NIVRS) is a common treatment in preterm infants with respiratory distress syndrome, either as primary support to avoid intubation, or as post-extubation support to facilitate further recovery.

- Different methods have been used to wean the preterm infants from NIVRS with varying success.

- The evidence regarding the optimal strategy for weaning is unclear.

\section{What this study adds?}

Gradual weaning of nasal continuous positive airway pressure possibly increases the chance of success of the first weaning attempt, but prolongs the weaning process.

- A stepdown strategy to nasal cannula accelerates the weaning process, but is associated with a longer duration of oxygen administration.

- Interval training shows no benefits and should probably not be applied in preterm infants.

post-extubation support and bronchopulmonary dysplasia. Spontaneous breathing can be supported non-invasively either by applying a continuous positive airway pressure (CPAP), or by providing positive pressure inflation breaths with an end-expiratory pressure (noninvasive intermittent positive pressure ventilation or NIPPV). In preterm infants, CPAP is typically applied using a device that controls proximal airway pressure, although nowadays also heated and humidified high-flow nasal cannula (HFNC) with flows between $2 \mathrm{~L} / \mathrm{min}$ and $8 \mathrm{~L} / \mathrm{min}$ is considered as CPAP. ${ }^{12}$

Weaning of premature infants from NIVRS to unsupported breathing is usually started as soon as stable conditions are reached. 
Weaning too quickly could result in an increased work of breathing (WOB) and a deterioration of respiratory function, which in turn could lead to a prolonged need for respiratory support and a prolonged hospital stay. ${ }^{3}$ Weaning too slowly, on the other hand, is associated with unnecessary exposure to respiratory support, and could therefore increase the risk of developing chronic lung disease (CLD) and/or retinopathy of prematurity (ROP). Some studies have suggested that a longer support with CPAP leads to improved structural lung growth resulting in better lung volumes. ${ }^{4}$ The optimal method to wean premature infants from CPAP and HFNC is still unclear, and methods used vary considerably between neonatal units. Recent reviews ${ }^{1245}$ concluded that there is not enough evidence to suggest an optimal strategy for weaning from CPAP or HFNC.

We conducted an updated systematic review and metaanalysis of randomised controlled trials (RCTs) to determine the risks and benefits of different strategies used for the withdrawal of CPAP and HFNC in preterm infants who are stable and may be ready for weaning.

\section{METHODS}

This systematic review and meta-analysis was conducted following a protocol that was registered in PROSPERO (CRD42019125327). MEDLINE, EMBASE, the Cochrane Central Register of controlled trials, CINAHL and Google were searched from inception to December 2019. The search terms included index terms (Mesh or Emtree) as well as free text words for "premature infant", "continuous positive airway pressure" or "high-flow nasal cannula" and "weaning".

Ongoing or unpublished trials were searched through trial registers and if needed by contacting the author of the study. The reference lists of retrieved articles were manually screened and studies were selected based on their title, abstract and method. Only studies in English, French, German, Spanish or Dutch were included. Eligibility criteria, study selection, risk of bias and quality of evidence assessments and statistical analysis are described in an online supplemental appendix 1.

The following CPAP systems were accepted for inclusion: (1) any mechanical device that is able to deliver a controlled continuous proximal airway pressure, such as a mechanical ventilator or an infant flow driver; (2) a bubble-CPAP system with underwater column to control proximal airway pressure; or (3) nasal cannula providing heated and humidified flow of gas at rate of at least $2 \mathrm{~L} /$ min, which has been shown to provide a positive pressure at the airway opening of $2-5 \mathrm{cmH}_{2} \mathrm{O}$. ${ }^{6}$ Trials had to either compare a specific weaning strategy with no weaning strategy or compare two different weaning strategies. Trials in which intermittent positive pressures were applied, such as (synchronised) NIPPV or bi-level CPAP, were excluded.

The prespecified primary outcome, time to successful weaning, was slightly adjusted after data extraction from a continuous to a dichotomous outcome, namely 'successful weaning at the first attempt' (ie, being successfully off NIVRS for at least 72 hours). Other main outcomes were the weaning strategy failure rate (ie, the need to restart the respiratory support after discontinuation or any failure to adhere to the predefined weaning strategy during the course of the study), and respiratory failure during the weaning process (ie, the need for endotracheal intubation and mechanical ventilation). Secondary outcomes were postmenstrual age (PMA) at successful wean (added post hoc), total duration of NIVRS, total duration of supplementary oxygen administration, total duration of hospitalisation, use of caffeine or other respiratory stimulants during weaning time, presence of air leak, presence of CLD, presence of nasal or facial injury and mortality during neonatal hospitalisation.

For the interventions, we considered any strategy that involved the stopping or gradual withdrawal of CPAP and/or HFNC. Possible weaning strategies were: (1) gradual weaning of proximal airway pressure for CPAP or flow rate for HFNC; (2) stepdown weaning, that is, switching from CPAP to either high-flow or low-flow nasal cannula (LFNC), or from HFNC to LFNC, based on prespecified criteria; (3) interval-based weaning that is, removing nasal CPAP or HFNC for short periods over 24 hours and gradually increasing the time off positive airway pressure based on prespecified criteria until the respiratory support is completely stopped. The complete and sudden discontinuation of support, independently of the level of pressure or flow was considered as control group. In order to assess the effects of each specific type of weaning strategy separately (stepdown vs gradual weaning vs interval training), trials were grouped by type of weaning strategy for analyses.

\section{RESULTS}

The search retrieved a total of 889 citations (figure 1). Following removal of duplicates and ineligible citations, we included 15 studies for the qualitative analysis of which 13 were eligible for the quantitative analysis. One trial $^{7}$ could not be included in the quantitative analysis because data could not be extracted in the required format.

A summary of the included trials is presented in online supplemental appendix 2. The trials investigated various weaning strategies of CPAP: (1) gradual weaning of CPAP pressure, $^{89}$ (2) stepdown from CPAP to a lower level of respiratory support, being either HFNC, LFNC or a combination of both, ${ }^{10-13}{ }^{14}$ (3) interval training where CPAP was cycled off with periods of either no support or a lower level of support, gradually increasing the time off until discontinuation of $\mathrm{CPAP}^{71015-19}$ and (4) a combination of the described methods. In most studies these strategies were compared with sudden discontinuation of CPAP, although some variation existed in how the control intervention was applied. Only one study made a direct comparison of two specific strategies: stepdown strategy versus gradual 


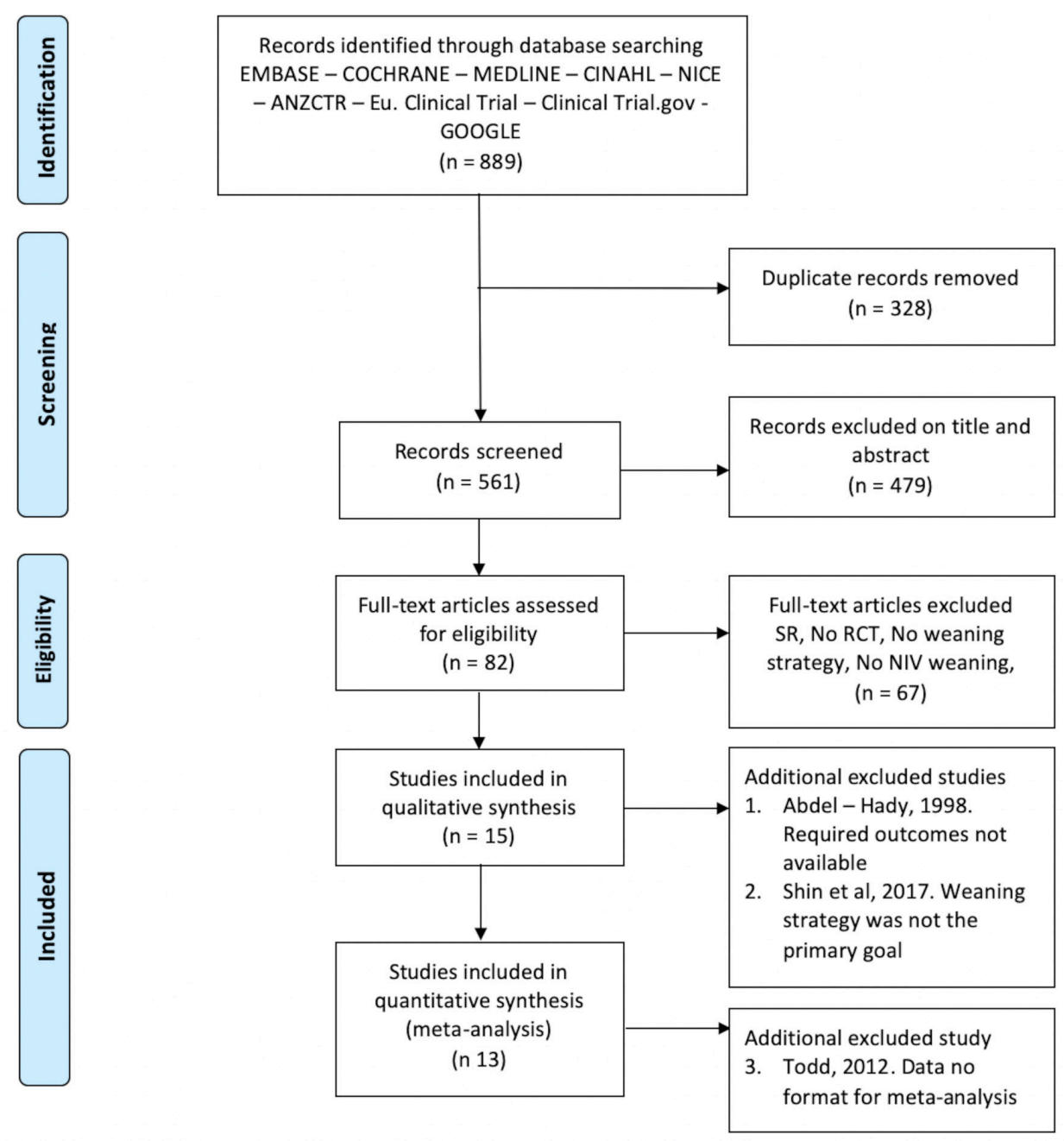

Figure 1 PRISMA flow diagram. Qualitative refers to qualitative assessment of the study methodology and quantitative is the number of studies included in the meta analysis. NIV, non-invasive ventilation; PRISMA, Preferred Reporting Items for Systematic Reviews and Meta-Analyses; RCT, randomised controlled trial; SR, systematic review.

pressure weaning. ${ }^{14}$ Three studies $^{7} 1018$ investigating interval training as weaning strategy, had two interventional arms, whereby the respiratory support during periods off CPAP varied from no support at all to LFNC with either 0.2 or $1.5 \mathrm{~L} / \mathrm{min},{ }^{18}$ or $\mathrm{HFNC}$ with $6 \mathrm{~L} / \mathrm{min}^{14}{ }^{14}$ In two trials with multiple interval training arms, ${ }^{10} 18$ we excluded the intervention group where during the pauses of CPAP nasal cannula with a higher flow was applied ${ }^{10 \mathrm{~b}} 18 \mathrm{~b}$ from the meta-analysis, because they were considered to be less consistent with the review question. All other comparisons were included in the meta-analyses. Readiness to wean was usually defined as a combination of a critical CPAP pressure, a low fractional inspired oxygen $\left(\mathrm{FiO}_{2}\right)$ and signs of clinical stability. Criteria for weaning failure were reported in all studies and showed good consistency across studies. Results of the risk of bias assessment of included studies are given in online supplemental appendix 1.

\section{Successful weaning at the first attempt and respiratory} failure during the weaning period

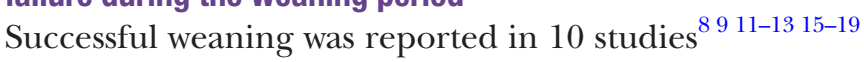
(figure 2). There was a non-significant trend towards an increased chance of successful weaning at the first attempt when CPAP pressure was gradually reduced as compared with abruptly stopped ( 2 trials, 422 infants, risk ratio (RR) 1.30 (95\% CI 0.93 to 1.83 )). No differences were found in the chance of successful weaning when a stepdown strategy ( 3 trials, 226 infants, RR 0.99 (95\% CI 0.85 to 1.15 )) or interval training ( 5 trials, 346 infants, RR 0.98 (95\% CI 0.85 to 1.15 )) was used compared with sudden weaning. There was no significant heterogeneity across trials in the meta-analysis.

The PMA in weeks at which the infant was successfully weaned was significantly higher with gradual CPAP weaning as compared with abrupt stopping of CPAP (2 trials, 422 infants, mean difference (MD) 0.93 weeks (95\% CI 0.19 to 1.67$)$ ). On the contrary, applying a stepdown strategy resulted in an almost 3-week reduction in PMA at successful weaning from CPAP as compared with abrupt stopping (2 trials, 118 infants, MD -2.70 weeks (95\% CI -3.87 to -1.52$)$ ) (figure 3 ). Of note, both trials did not find a significant difference in the PMA when infants came off any respiratory support (CPAP, HFNC 


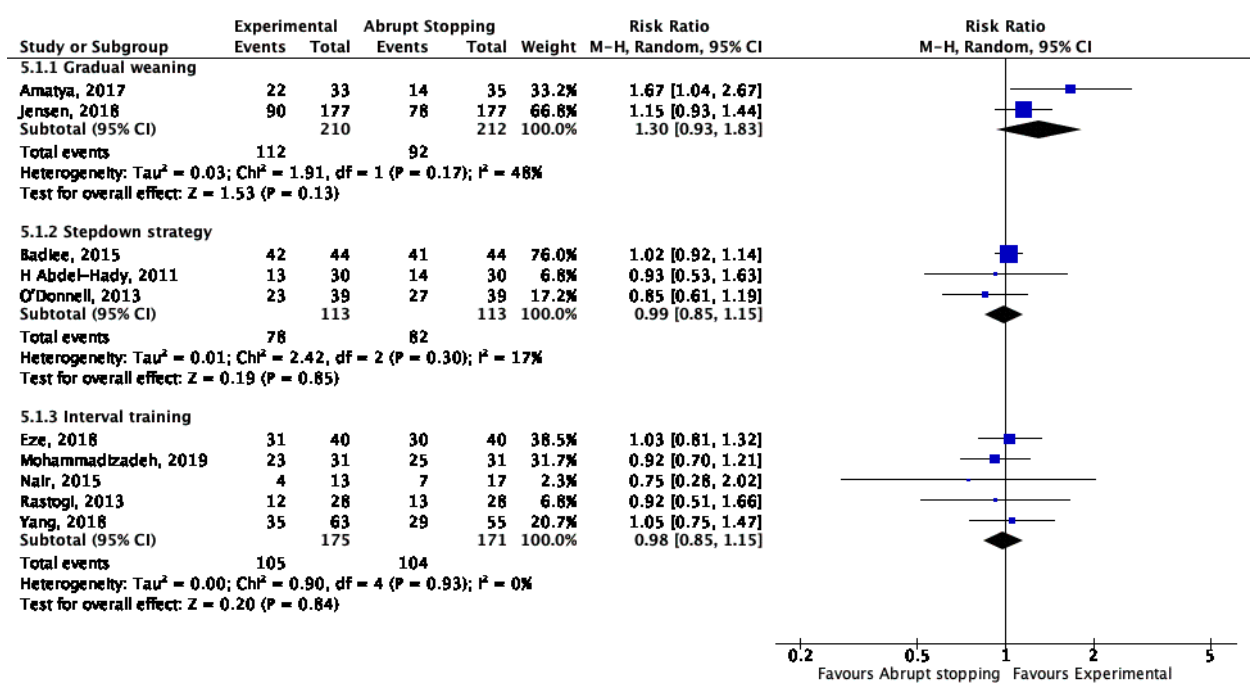

or oxygen). For interval training, marked heterogeneity existed across studies for this outcome $\left(I^{2}=87 \%\right)$, with studies showing earlier weaning ${ }^{17} 18$ as well as delayed weaning. ${ }^{10}$

Respiratory failure during the weaning period was only reported in three trials. ${ }^{101314}$ Badiee et al ${ }^{13}$ reported more respiratory failure when CPAP was abruptly stopped compared with a stepdown approach $(4 / 44$ vs $0 / 44$, $\mathrm{p}=0.05)$. In the study by Soonsawad et al, ${ }^{14}$ comparing a stepdown strategy to HFNC and further weaning of flow to a strategy of weaning of CPAP pressure, only 1 infant (from the stepdown strategy group) out of the 101 included infants had respiratory failure.

\section{Weaning failure during the course of the study}

There is no significant difference in weaning failure rate when a stepdown strategy is compared with abrupt stopping of CPAP ( 4 trials, 327 infants, RR 1.25 (95\% CI 0.79 to 1.97$)$ ) (online supplemental appendix 3$).{ }^{11-14}$

\section{Total duration of NIVRS}

There was a modest, but statistically significant increase in the duration of CPAP treatment when CPAP was gradually decreased as compared with abruptly stopped (2 trials, 422 infants, MD 1.52 days (95\% CI 0.73 to 2.30$)$ ) (online supplemental appendix 4).

The two studies (90 infants) comparing a stepdown strategy to HFNC (flow of $2 \mathrm{~L} / \mathrm{min}$ or $6 \mathrm{~L} / \mathrm{min}$ ) with abrupt stopping of CPAP both showed a significant reduction in CPAP duration but the effect size differed markedly: -3.60 days $(95 \%$ CI -6.98 to -0.22$)$ for the study by Abdel-Hady et $a l^{11}$ versus -17.7 days $(95 \%$ CI -21.00 to -14.40 ) for the study by Tang et al. ${ }^{10}$ Interval training resulted in a significant increase in duration of NIVRS compared with the abrupt stopping of CPAP (4 trials, 240 infants 1.66 days (95\% CI -0.86 to 2.46$)$ ) .

\section{Total duration of oxygen supplementation}

As compared with abrupt stopping CPAP, both gradual weaning (2 trials, 422 infants, MD 1.45 days $(95 \% \mathrm{CI}$

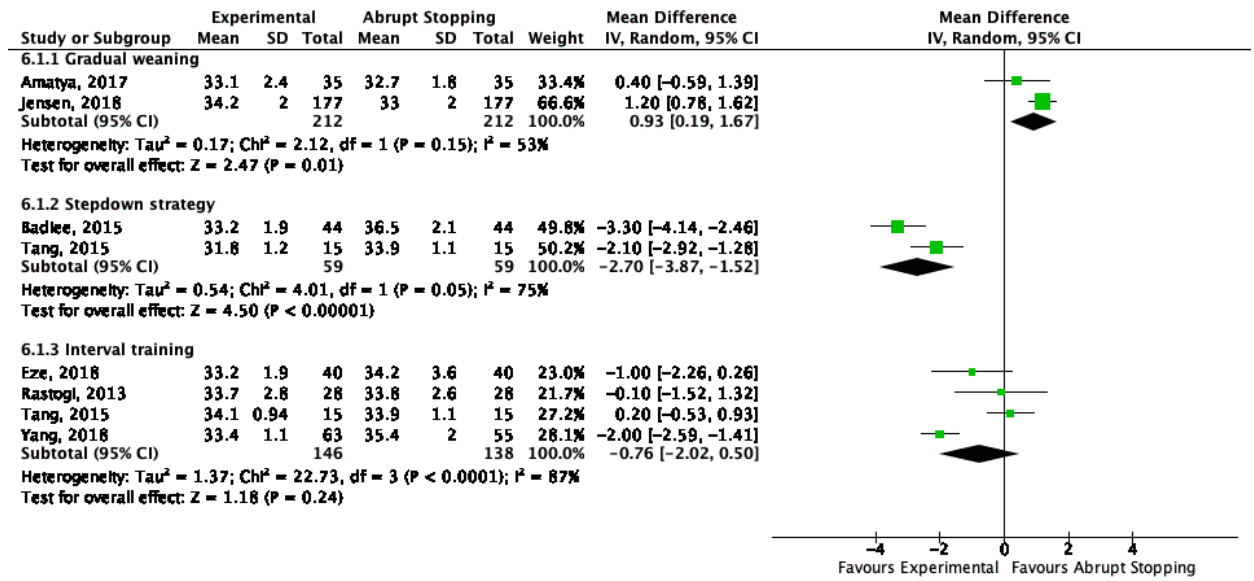

Figure 3 Postmenstrual age in weeks at the first successful weaning trial (gradual weaning, stepdown strategy and interval training vs abrupt stopping). 
0.38 to 2.53$)$ ) as well as a stepdown strategy (2 trials, 148 infants, MD 7.80 days (95\% CI 5.31 to 10.28$)$ ) resulted in a significant increase in the total duration of oxygen supplementation (online supplemental appendix 5). For interval training, no significant effect of interval training on the duration of oxygen supplementation was found (3 trials, 236 infants, $\mathrm{MD}-0.03$ days ( $95 \% \mathrm{CI}-0.16$ to 0.10$)$ ).

\section{Weaning of CPAP and use of caffeine}

Five $^{8-1115}$ trials reported on the use of caffeine and four ${ }^{11} 131819$ on the use of xanthine for the treatment of apnoeas of prematurity as baseline therapy. There was no significant relationship between the need of caffeine or xanthine and a specific weaning strategy of CPAP in any of the trials.

\section{Length of hospital stay}

Length of hospital stay (online supplemental appendix 6) was reported in 10 10-11 13-15 17-19 studies. A strategy of gradual pressure weaning had no significant effect on the duration of hospitalisation (2 trials, 422 infants, MD 0.26 days (95\% CI -8.44 to 8.96$)$ ). Using a stepdown strategy resulted in a significantly earlier discharge as compared with abrupt stopping ( 3 trials, 178 infants, MD -3.51 days (95\% CI -4.04 to -2.98$)$ ). For interval training, the metaanalysis showed a not significant increase in the length of hospital stay ( 5 trials, 346 infants, MD 1.26 days $(95 \%$ CI -1.88 to 4.40$)$ ).

\section{Adverse events}

For adverse events, only four studies ${ }^{10} 111419$ reported air leaks and/or facial/nasal injuries. Soonsawad et al showed less nasal trauma in the HFNC compared with the CPAP group ( $20 \%$ vs $42 \%$ ). In Tangs' study, there was no significant difference in nasal injury between the weaning groups. Presence of air leak was only described by Abdel-Hady et al and Mohammadizadeh et al. In both RCTs there was no difference between the abrupt stopping group compared with the stepdown strategy and interval training.

\section{Chronic lung disease}

The effect of using a certain CPAP weaning strategy on the risk of CLD, defined as the need of respiratory support or oxygen need at 36 weeks' PMA, was reported in 12 studies. For none of the weaning strategies a significant effect was seen on the risk of CLD (figure 4). This finding was consistent across all trials.

\section{DISCUSSION}

This systematic review and meta-analysis identified 13 RCTs where different weaning strategies were studied for successful weaning of nasal CPAP in preterm infants. Except for one study, ${ }^{14}$ weaning strategies were always compared with the sudden discontinuation of CPAP. Three categories of weaning strategies could be distinguished: (1) gradual weaning of CPAP pressure, (2) stepping down from CPAP to a lower level of respiratory support and (3) interval training with a prespecified schedule of cycling off CPAP. Both the short-term success or failure of the different strategies (primary outcomes) as well as the more clinically relevant longer-term effects on CLD or ROP (secondary outcomes) were assessed.

With gradual pressure wean, which was addressed by the largest included trial, ${ }^{9}$ infants were possibly more successful in their first attempt to be weaned off CPAP as compared with sudden discontinuation, but they remained on CPAP for 1 week longer in terms of their PMA. This prolonged CPAP treatment did not affect the duration of hospitalisation. A recent study suggested that extended CPAP application on itself may have a stimulatory effect on lung growth, resulting in larger functional residual capacity (FRC). This positive effect of CPAP on FRC development may in fact explain the higher

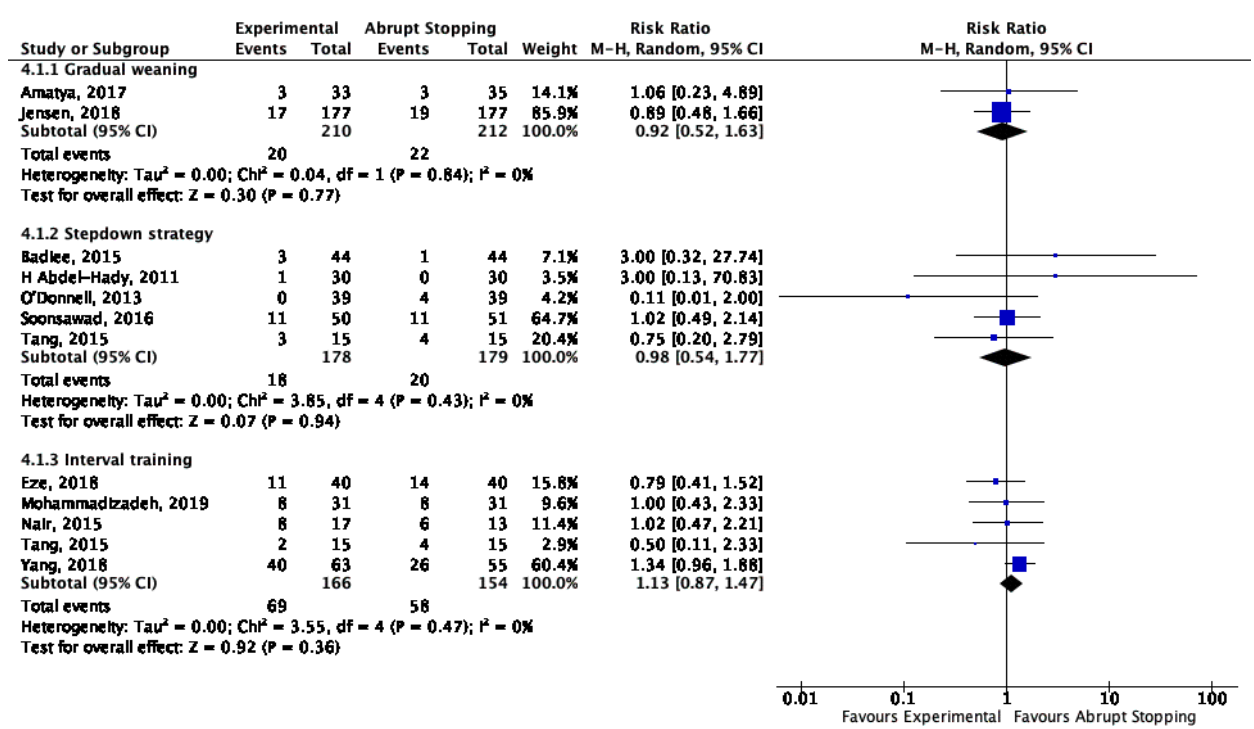

Figure 4 Chronic lung disease at 36 weeks' gestation (gradual weaning, stepdown strategy and interval training vs abrupt stopping). 
success rate of a first weaning trial. The higher PMA at the weaning attempt could of course by itself be responsible for the higher success rate. The main challenge with this strategy is knowing the optimal CPAP pressure for each individual infant at each individual time point of the disease. An adequate positive airway pressure is essential to maintain an optimal FRC, which is reflected by adequate oxygenation, minimal WOB and haemodynamic stability. ${ }^{20-23}$ Unfortunately, there is only limited evidence to suggest a single approach to initiate or adjust the CPAP level in preterm infants recovering from RDS.

Stepping down from CPAP to a lower level of support, either HFNC or LFNC, clearly reduced the time on CPAP as compared with sudden discontinuation. Infants were on average 2.7 weeks younger in PMA when they were successfully weaned off CPAP, and success rates of the first attempt were similar. However, infants remained significantly longer on oxygen supplementation (on average 1 week). Possible explanations are the lack of beneficial effects of CPAP on lung development in the group where CPAP was stopped earlier, or the lack of focus on continued strict weaning once infants were on nasal cannula because it causes less discomfort to the infant. Although this was not associated with a prolonged hospital stay or an increased risk of CLD, it is uncertain whether or not it could increase the risk of developing ROP. In the study by Yang $e t a l,{ }^{18}$ infants in the group of $100 \%$ oxygen LFNC $(0.2 \mathrm{~L} / \mathrm{min})$ had a significantly increased risk of ROP as compared with the other two groups (52\% vs $38 \%$ in the CPAP group and $44 \%$ in the nasal cannula group with air flow, $\mathrm{p}<0.05)$. This finding was not confirmed in the other four studies ${ }^{10141617}$ that reported on the incidence of ROP.

In most of the studies investigating the stepdown strategy, infants were switched from CPAP to HFNC although flows varied between $2 \mathrm{~L} / \mathrm{min}$ and $6 \mathrm{~L} / \mathrm{min}$. Nasal CPAP provides a consistent positive pressure at the proximal airway which is monitored continuously in order to keep the alveoli open and maintain an optimal FRC. ${ }^{24-27}$ Studies have demonstrated that flows as low as $2 \mathrm{~L} / \mathrm{min}$ can generate a positive pressure up to $6 \mathrm{cmH}_{2} \mathrm{O}$, but also that this pressure is highly variable. ${ }^{6}{ }^{1828-30}$ Inconsistent pressure generation during HFNC could lead to micro-atelectasis, contributing to a prolonged need of respiratory support and oxygen need. HFNC has become commonly used alternative for nasal CPAP in neonatal units, mostly because of the comfort it offers to the infant and parents (less nasal trauma, ease of care, facilitating infant-parent bonding and kangaroo care). ${ }^{31-33}$ However, studies on how to wean HFNC are completely lacking. ${ }^{30}$

Interval training, although most frequently studied, does not seem to offer any benefit as compared with sudden discontinuation of CPAP. It was not associated with a higher success rate of the first weaning attempt, and infants were weaned off CPAP at a similar PMA, although for the latter outcome significant betweenstudy heterogeneity existed. It is likely that the interval training schedule itself has a major impact on its success.
In some trials, respiratory support during times off CPAP was completely removed or restricted to an LFNC. This could have resulted in intermittent de-recruitment of lung volume, and, hence, to increased WOB. ${ }^{34}{ }^{35}$ Intermittent withdrawal of positive airway pressure during interval training may be detrimental for the development of immature lungs.

An important factor in the weaning process and in the success of a specific strategy is undoubtedly the way infants are being assessed to be ready or not for (further) weaning. In all studies, clear readiness-to-wean criteria were defined in the protocol. Besides a minimally required level of CPAP pressure and $\mathrm{FiO}_{2}$, those criteria also consisted of clinical signs of respiratory stability, such as 'WOB' or 'chest retractions'. The clinical assessment of an infant's respiratory condition requires committed and trained nursing staff. It is known that the clinical expertise of the nursing staff is an important factor determining CPAP success. ${ }^{27}{ }^{36}$ Probably, it is of equal importance during the weaning phase of CPAP. Also, readiness-to-wean should be assessed in a very consistent way. Therefore, it is important that each unit develops its own specific weaning protocol and invests in adequate training of nursing staff.

The strengths of this systematic review are the comprehensiveness of the literature search and the fact that a prespecified, strict methodology, published in PROSPERO, was followed. In addition, the majority of the included trials in this review were published in the past 5 years representing well current clinical practice about CPAP weaning.

This review has also some limitations. Some predefined outcomes required minor adjustments after data extraction. For some studies, imputation was required in order to have the data in the correct format. Especially in meta-analyses with only few studies, this could have an impact on the meta-analysis result. We were unable to include two RCTs ${ }^{37}{ }^{38}$ which were published only in abstract form, even after having contacted the authors. For some of the trials, the data could not be obtained in the correct format for meta-analysis, even after contacting the authors, making it impossible to include those studies in the meta-analyses. Due to the fact that the interventions were technically very difficult or even impossible to blind for caregivers, all included trials are at risk of performance bias. Finally, not all factors that possibly modify the effects of a weaning strategy (eg, severity of RDS, use of antenatal steroids, use of device and interfaces) could be taken into account in this review.

\section{CONCLUSION}

This systematic review and meta-analysis showed that a weaning strategy of progressive reduction of CPAP pressure possibly increases the chances of success at first weaning attempt, but that the weaning process takes more time and discontinuation comes at a later PMA. Stepping down from CPAP to an HFNC shortens the 
duration of CPAP treatment but is associated with a longer duration of oxygen administration. Whether one strategy is superior to another should be further investigated in a head-to-head comparative study. Studies on how to wean HFNC further are currently lacking. No major benefits were found for a weaning strategy based on interval training. None of the weaning strategies had any effect on the development of CLD.

Neonatal units should make their own specific weaning protocol with prespecified readiness-to-wean criteria and provide adequate training for nursing staff, so that CPAP weaning is consistent and transparent within a certain unit.

Future studies are needed on CPAP pressure during the weaning process to maintain optimal lung volume at all times, on the objective assessment of readiness to be weaned and on possible strategies to safely wean HFNC.

Contributors BvD had full access to all the data in the review and takes responsibility for the integrity of the data and the accuracy of the data analysis. BvD, FVG, JL, CvD and FC contributed to study concept or design, administrative, technical or material support. BvD and FC contributed to drafting of the article and statistical analysis. All authors contributed to critical revision of the article, the acquisition, analysis or interpretation of data.

Funding The authors have not declared a specific grant for this research from any funding agency in the public, commercial or not-for-profit sectors.

Competing interests None declared.

Patient consent for publication Not required.

Provenance and peer review Not commissioned; externally peer reviewed.

Data availability statement All data relevant to the study are included in the article or uploaded as supplemental information. For supplemental information, send email to author.

Supplemental material This content has been supplied by the author(s). It has not been vetted by BMJ Publishing Group Limited (BMJ) and may not have been peer-reviewed. Any opinions or recommendations discussed are solely those of the author(s) and are not endorsed by BMJ. BMJ disclaims all liability and responsibility arising from any reliance placed on the content. Where the content includes any translated material, BMJ does not warrant the accuracy and reliability of the translations (including but not limited to local regulations, clinical guidelines, terminology, drug names and drug dosages), and is not responsible for any error and/or omissions arising from translation and adaptation or otherwise.

Open access This is an open access article distributed in accordance with the Creative Commons Attribution Non Commercial (CC BY-NC 4.0) license, which permits others to distribute, remix, adapt, build upon this work non-commercially, and license their derivative works on different terms, provided the original work is properly cited, appropriate credit is given, any changes made indicated, and the use is non-commercial. See: http://creativecommons.org/licenses/by-nc/4.0/.

ORCID iD

Brenda van Delft http://orcid.org/0000-0001-7935-1599

\section{REFERENCES}

1 Farley RC, Hough JL, Jardine LA, et al. Strategies for the discontinuation of humidified high flow nasal cannula (HHFNC) in preterm infants. Cochrane Database Syst Rev 2015;87:1-12.

2 Abdel-Hady H, Shouman B, Nasef N. Weaning preterm infants from continuous positive airway pressure: evidence for best practice. World J Pediatr 2015;11:212-8.

3 Gizzi C, Massenzi L, Pattumelli MG, et al. Weaning of infants from non invasive ventilation. Acta Biomed 2014;85:15-19.

4 Amatya S, Rastogi D, Bhutada A, et al. Weaning of nasal CPAP in preterm infants: who, when and how? A systematic review of the literature. World J Pediatr 2015;11:7-13.

5 Jardine LA, Inglis GDT, Davies MW, et al. Strategies for the withdrawal of nasal continuous positive airway pressure (nCPAP) in preterm infants. Cochrane Database Syst Rev 2011;165:1-22.
6 Wilkinson DJ, Andersen CC, Smith K, et al. Pharyngeal pressure with high-flow nasal cannulae in premature infants. J Perinatol 2008;28:42-7.

7 Todd DA, Wright A, Broom M, et al. Methods of weaning preterm babies $<30$ weeks gestation off CPAP: a multicentre randomised controlled trial. Arch Dis Child Fetal Neonatal Ed 2012;97:F236-40.

8 Amatya S, Macomber M, Bhutada A, et al. Sudden versus gradual pressure wean from nasal CPAP in preterm infants: a randomized controlled trial. J Perinatol 2017;37:662-7.

9 Jensen CF, Sellmer A, Ebbesen F, et al. Sudden vs pressure wean from nasal continuous positive airway pressure in infants born before 32 weeks of gestation: a randomized clinical trial. JAMA Pediatr 2018;172:824-31.

10 Tang J, Reid S, Lutz T, et al. Randomised controlled trial of weaning strategies for preterm infants on nasal continuous positive airway pressure. BMC Pediatr 2015;15:1-8.

11 Abdel-Hady H, Shouman B, Aly H. Early weaning from CPAP to high flow nasal cannula in preterm infants is associated with prolonged oxygen requirement: a randomized controlled trial. Early Hum Dev 2011;87:205-8.

12 O'Donnell SM, Curry SJ, Buggy NA, et al. The NOFLO trial: low-flow nasal prongs therapy in weaning nasal continuous positive airway pressure in preterm infants. J Pediatr 2013;163:79-83.

13 Badiee Z, Eshghi A, Mohammadizadeh M. High flow nasal cannula as a method for rapid weaning from nasal continuous positive airway pressure. Int J Prev Med 2015;6:33-6.

14 Soonsawad S, Tongsawang N, Nuntnarumit P. Heated humidified high-flow nasal cannula for weaning from continuous positive airway pressure in preterm infants: a randomized controlled trial. Neonatology 2016;110:204-9.

15 Rastogi S, Wong W, Gupta A, et al. Gradual versus sudden weaning from nasal CPAP in preterm infants: a pilot randomized controlled trial. Respir Care 2013;58:511-6.

16 Nair V, Swarnam K, Rabi Y, et al. Effect of nasal continuous positive airway pressure (nCPAP) cycling and continuous nCPAP on successful weaning: a randomized controlled trial. Indian J Pediatr 2015;82:787-93.

17 Eze N, Murphy D, Dhar V, et al. Comparison of sprinting vs nonsprinting to wean nasal continuous positive airway pressure off in very preterm infants. J Perinatol 2018;38:164-8.

18 Yang C-Y, Yang M-C, Chu S-M, et al. A randomized pilot study comparing the role of PEEP, $\mathrm{O}_{2}$ flow, and high-flow air for weaning of ventilatory support in very low birth weight infants. Pediatr Neonatol 2018:59:198-204.

19 Mohammadizadeh M, Badiei Z, Choopani R, et al. Sudden complete versus gradual weaning from nasal continuous positive airway pressure in preterm neonates: a randomized controlled trial. IJN 2019;10:9-16.

20 Rastogi S, Rajasekhar H, Gupta A, et al. Factors affecting the weaning from nasal CPAP in preterm neonates. Int $J$ Pediatr 2012;2012:1-7.

21 Fathi O, Brown A. Non-Invasive ventilation of the neonate. Intechopen 2017:61-80.

22 Maffei G, Gorgoglione S, Vento G. Noninvasive ventilation: systematic approach and new perspectives for preterm infants. $J$ Clin Neonatol 2017;6:135-43.

23 Lam R, Schilling D, Scottoline B, et al. The effect of extended continuous positive airway pressure on changes in lung volumes in stable premature infants: a randomized controlled trial. J Pediatr 2020;217:66-72.

24 Hooper SB, te Pas AB, Lewis RA, et al. Establishing functional residual capacity at birth. Neoreviews 2010;11:e474-83.

25 Gerhardt T, Reifenberg L, Hehre D, et al. Functional residual capacity in normal neonates and children up to 5 years of age determined by a N2 washout method. Pediatr Res 1986;20:668-71.

26 Bhat L, Khanijo K, et al. Can higher PEEP and FiO2 with bubble CPAP reduce need for invasive ventilation in preterm babies with respiratory distress syndrome? J Neonatal Biol 2014;05:1-3.

27 Collins CL, Holberton JR, König K. Comparison of the pharyngeal pressure provided by two heated, humidified high-flow nasal cannulae devices in premature infants. J Paediatr Child Health 2013;49:554-6.

28 Ed Liew Z, Fenton AC, Harigopal S, et al. Physiological effects of high-flow nasal cannula therapy in preterm infants. Arch Dis Child Fetal Neonatal Ed 2020;105:87-93.

29 Kubicka ZJ, Limauro J, Darnall RA. Heated, humidified high-flow nasal cannula therapy: yet another way to deliver continuous positive airway pressure? Pediatrics 2008;121:82-8.

30 Acke E. Weaning strategies of heated and humidified high-flow nasal cannulae - a form of non-invasive ventilation in preterm infants. $\mathrm{KU}$ Leuven, 2019: 3-24. 
31 Roberts CT, Hodgson KA. Nasal high flow treatment in preterm infants. Matern Health Neonatol Perinatol 2017;3:1-9.

32 Glackin SJ, O'Sullivan A, George S, et al. High flow nasal cannula versus $\mathrm{nCPAP}$, duration to full oral feeds in preterm infants: a randomised controlled trial. Arch Dis Child Fetal Neonatal Ed 2017;102:F329-32.

33 Hoffman SB, Terrell N, Driscoll CH, et al. Impact of high-flow nasal cannula use on neonatal respiratory support patterns and length of stay. Respir Care 2016;61:1299-304.

34 Ellwein Fix L, Khoury J, Moores RR, et al. Theoretical open-loop model of respiratory mechanics in the extremely preterm infant. PLoS One 2018;13:e0198425-21.
35 Mols G, Priebe H-J, Guttmann J. Alveolar recruitment in acute lung injury. Br J Anaesth 2006;96:156-66.

36 Gentle SJ, Ambalavanan N, Carlo WA. Oxygen saturation histograms predict nasal continuous positive airway pressure-weaning success in preterm infants. Pediatr Res 2020;88:637-41.

37 Singh* SD, Clarke P, Bowe L, et al. Nasal CPAP weaning of VLBW infants: is decreasing CPAP pressure or increasing time off the better strategy? results of a randomised controlled trial. Early Hum Dev 2007;83:130-1.

38 Soe A, Hodgkinson J, Jani B, et al. Nasal continuous positive airway pressure weaning in preterm infants. Eur $J$ Peadiatr 2006;165:48-9. 